\title{
Efficacy of immune checkpoint inhibitors and age in cancer patients
}

\author{
Xuan-zhang Huang ${ }^{1}$, Peng Gao'1, Yong-xi Song1', Jing-xu Sun1', Xiao-wan Chen'1, Jun-hua \\ Zhao $^{1}$ \& Zhen-ning Wang*,1 (iD \\ ${ }^{1}$ Department of Surgical Oncology \& General Surgery, Key Laboratory of Precision Diagnosis \& Treatment of Gastrointestinal \\ Tumors, Ministry of Education, The First Affiliated Hospital of China Medical University, 155 North Nanjing Street, Heping District, \\ Shenyang City 110001 , China \\ *Author for correspondence: Tel.: +86 248328 3556; Fax: +86 242270 3578; josieon826@sina.cn
}

\begin{abstract}
Aim: To evaluate the impact of age on the efficacy of immune checkpoint inhibitors (ICI) in cancer patients. Materials \& methods: The primary outcomes included overall survival (OS) and progression-free survival (PFS). Subgroup, meta-regression analysis and within-trial interaction HR were conducted. Results: A total of 34 studies containing 20,511 cancer patients were included. ICl could improve the OS and PFS in patient aged $<65$ and $\geq 65$ years. Patients aged $<75$ years treated with $\mathrm{ICl}$ also had favorable OS and PFS compared with the control groups. Conclusion: ICI has comparable efficacy in cancer patients aged $<65$ and $\geq 65$ years. Cancer patients aged $\geq 75$ years need more attention in the future clinical trials.
\end{abstract}

First draft submitted: 22 July 2019; Accepted for publication: 24 April 2020; Published online: 7 May 2020

Keywords: age $\bullet$ immune checkpoint inhibitors $\bullet$ immunotherapy $\bullet$ overall survival $\bullet$ progression-free survival

Programmed cell death protein-1 (PD-1), programmed cell death-ligand 1 (PD-L1) and cytotoxic T lymphocyteassociated antigen 4 (CTLA-4) are the predominant and most well-studied immune checkpoints associated with immune escape by tumor cells [1,2]. The immune checkpoint inhibitors (ICI) against PD-1, PD-L1 and CTLA-4 have been shown to exhibit improved efficacy, lower toxicity and higher safety profiles compared with traditional cytotoxic agents for cancer treatment [3]. Thus, immunotherapies based on ICI have become an attractive treatment option and changed the therapeutic landscape for several advanced malignancies.

Cancer is predominantly a disease affecting older adults, with a significantly higher incidence in patients aged $\geq 65$ years [4]. Although older adults comprise the majority of patients diagnosed with cancer in the 'real world', older adults continue to be under-represented in clinical studies evaluating ICI immunotherapies since older patients tend to exhibit a higher number of comorbidities and less favorable performance status; therefore, these patients are frequently excluded from clinical studies [5]. In addition, the age-related decline in immune function leads to immune dysregulation in both cellular and humoral responses, which may be an important reason for a higher incidence of cancer with age [6-8]. Moreover, the phenomenon of gradual aging and deterioration of immune function with age is termed immunosenescence [6,7]. Age-related alterations in the immune system primarily occur with regards $\mathrm{T}$ cell-mediated immune function, which is a crucial aspect of immunosenescence [6]. Importantly, $\mathrm{T}$ cells are the major antitumor effector cells for ICI immunotherapies and some researchers consider ICI immunotherapies to have reduced efficacy in older cancer patients due to the negative effect induced by immunosenescence. Therefore, it is inappropriate to manage older cancer patients simply using the clinical evidence extrapolated from studies of younger cancer patients.

A previous meta-analysis by Wu et al. only found that ICI immunotherapies significantly improved survival in both patient groups of $<65$ and $\geq 65$ years, but did not evaluate the patient population aged $\geq 75$ years [9]. Li et al. reported that ICI immunotherapies had comparable benefit in both of the younger $(<65$ years) and older cancer patients ( $\geq 65$ years) but did not prolong survival in patients aged $\geq 75$ years [10]. Interestingly, a melanoma-specific meta-analysis showed that melanoma patients aged $\geq 75$ years would benefit more from ICI immunotherapies compared with younger patients, which was opposite with the possible negative impact of immunosenescence on 
ICI efficacy [11]. Nevertheless, the number of included studies in these meta-analyses was limited. Thus, the result regarding the efficacy of ICI immunotherapies in older cancer patients still remains controversial.

The purpose of this study was to explore the efficacy of ICI immunotherapies in both younger and older cancer patients ( $<65$ vs $\geq 65$ years and $<75$ vs $\geq 75$ years).

\section{Materials \& methods}

\section{Literature search}

The PubMed and Embase databases were searched for Phase II and III randomized controlled trials (RCT) that evaluated the efficacy of ICI immunotherapies in younger and older cancer patients (up to February 2019). We also performed a literature search through the American Society of Clinical Oncology and European Society of Medical Oncology conference databases. The search terms consisted of 'nivolumab', 'pembrolizumab', 'avelumab', 'atezolizumab', 'lambrolizumab', 'pidilizumab', 'durvalumab', 'ipilimumab', 'tremelimumab', 'PD-1', 'PD-L1', 'programmed death protein 1', 'programmed death ligand 1', 'CTLA-4', 'cytotoxic T-lymphocyte associated protein 4', 'cytotoxic T-lymphocyte associated antigen 4', 'immune checkpoint inhibitor', 'randomised trial', 'randomized trial', 'randomised study' and 'randomized study'. Furthermore, we manually searched the reference lists of the relevant studies and reviews for other potential studies.

\section{Inclusion criteria}

The eligibility criteria for the Phase II or III RCTs to be included in this meta-analysis were as follows: participants: patients who were diagnosed with solid cancer and aged 18 years or older; intervention: ICIs (PD-1, PD-L1 or CTLA-4 inhibitors) were administered alone or in combination with another ICIs/chemotherapeutic agents, irrespective of the duration and dosage; comparison: the control group was treated with either a placebo or other non-ICI agents; outcome: the two primary outcomes consisted of the overall survival (OS) and progression-free survival (PFS) according to different age groups (i.e., $<65, \geq 65,<75$ and $\geq 75$ years) and the hazard ratio (HR) and corresponding $95 \%$ CI could be extracted. Moreover, we included the most recent study if several duplicated studies were identified based on the use of the same patient population.

\section{Exclusion criteria}

The exclusion criteria consisted of: animal or in vitro studies; cohort, case-control studies or Phase I trials; the outcome measures could not be extracted from the published data; the therapeutic regimen of the control group contained ICI or other nonspecific immunological agents; and duplicate studies based on the same population.

\section{Data extraction \& quality assessment}

Two authors (X Huang and P Gao) reviewed the eligible studies and independently extracted the data. For each study, the following data were extracted: first author, publication year, trial name, trial phase, study design, cancer type, therapy line, ICI drug, treatment regimens, sample size, age, follow-up time and outcome measures. The quality of the included studies was evaluated using the Jadad scale, which was based on three aspects of the study: randomization; blinding; and withdrawals/dropouts [12]. Any disagreements regarding the data extraction and quality assessment were resolved by discussion.

\section{Statistical analysis}

The primary outcomes consisted of the clinical efficacy (OS and PFS) of ICI immunotherapies between different age groups (i.e., $<65, \geq 65,<75$ and $\geq 75$ years), measured by $\mathrm{HR}$ with $95 \% \mathrm{CI}$. We used a random-effect model to pool the HRs because the random-effect model could obtain more conservative results than the fixed-effect model, considering the heterogeneity among multicenter clinical studies [13]. The overall analysis was performed by including all eligible studies and a subgroup analysis was performed by stratifying the data by cancer type (nonsmall-cell lung cancer, melanoma, renal-cell carcinoma, small-cell lung cancer, gastric cancer/gastro-esophageal junction cancer and head-and-neck squamous cell carcinoma), therapy line (line 1 and line $\geq 2$ ) and ICI drug (PD-1/PD-L1, CTLA-4, PD-1 and ICI plus chemotherapy). The heterogeneity among the studies was evaluated using a Cochran Q test and $I^{2}$ statistics [14]. Publication bias was evaluated using Begg's and Egger's tests [15,16].

We used a meta-regression analysis to assess the impact of age on the efficacy of ICI immunotherapies in cancer patients. Furthermore, we used the within-trial interaction $\mathrm{HR}$ (ratio of the $\mathrm{HR}$ in patients aged $<65$ and $\geq 65$ years; 


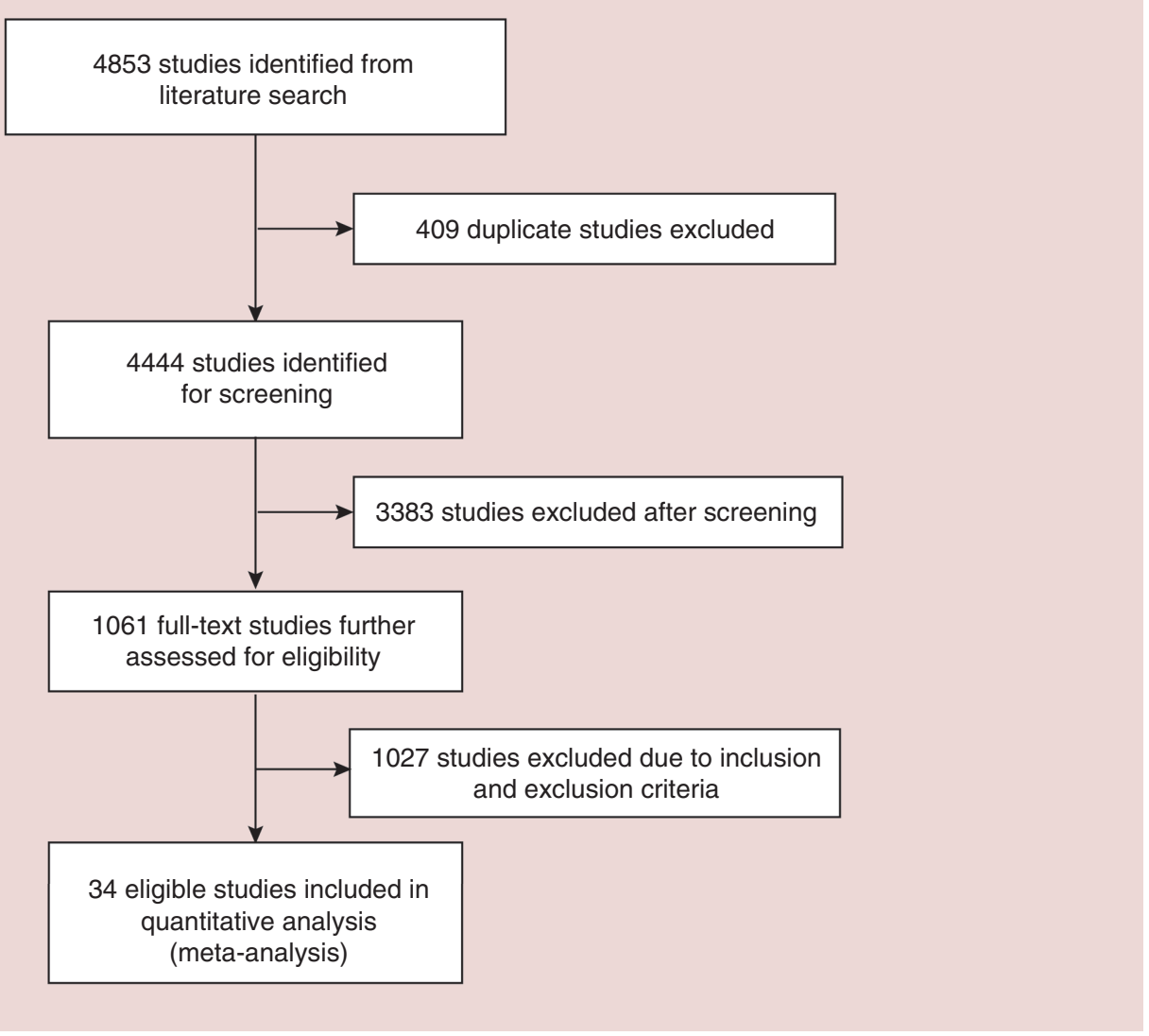

Figure 1. Literature search and study selection.

and $<75$ and $\geq 75$ years) to evaluate the differences in the efficacy of ICI immunotherapies between different age groups ( $<65$ vs $\geq 65$ years and $<75$ vs $\geq 75$ years) [17].

All statistical analyses were performed using Stata software version 12.0 (Stata Corporation, TX, USA). A two-sided p-value of $<0.05$ was considered statistically significant.

\section{Results}

\section{Study selection \& associated characteristics}

A total of 4853 studies were identified from our literature search, of which 409 studies were excluded due to duplications. A total of 3383 studies were excluded after reviewing the titles and abstracts and 1061 full-text studies were further assessed for eligibility. There were 1027 studies that were excluded based on the inclusion and exclusion criteria. Finally, 34 eligible studies were included in the quantitative analysis [18-51]. Figure 1 shows a detailed flowchart of the study selection.

The 34 included studies were published between 2010 and 2018, of which 21 (62\%) studies were published in 2017 and 2018. A total of 20,511 patients (mean: 603; median with range: 565 [272-1033]) were included in this meta-analysis. There were 32 Phase III RCTs [18-32,34-40,42-51] and one of each Phase IIb and II/III RCTs [33,41]. Of the eligible studies, 13 studies involved patients with non-small-cell lung cancer [18,21,25,26,29,31,32,35,36,39,41,46,47]; seven studies of patients with melanoma [23,27,43,44,49-51]; three studies of patients with gastric or gastro-esophageal junction cancer [19,30,34]; two studies each for patients with renal-cell cancer [22,45], small-cell lung cancer [24,40]; prostate cancer [38,48] and head-and-neck squamous cell carcinoma [28,42]; and one study each for breast cancer [20], malignant mesothelioma [33] and urothelial carcinoma [37] patients. Of the 20,511 patients, 7740 (38\%) patients had non-small-cell lung cancer and $4215(21 \%)$ had melanoma. In terms of the ICI drug, 24 studies used anti-PD1/PD-L1 inhibitors [18-21,23,24,26-32,34,36,37,39,41-47], eight studies used anti-CTLA-4 inhibitors [33,35,38,40,48-51] and two studies used anti-PD-1/PD-L1 inhibitors plus anti-CTLA-4 inhibitors [22,25]. There were 17 studies each 
evaluating the efficacy of ICI as a first-line therapy $[18,20-22,24-27,31,35,36,38-40,43,49,50]$ and second or more line therapies [19,23,28-30,32-34,37,41,42,44-48,51], respectively. A total of 11,162 patients were aged $<65$ years and 7865 patients were aged $\geq 65$ years. There were 13 studies that reported an age group of $\geq 75$ years with a total of 630 patients $[18,22,25,28-30,35,40,42,43,45-47]$. The baseline characteristics of the included studies are summarized in Table 1.

\section{Overall survival \& cut-off age of 65 years}

Our results indicated that ICI immunotherapies significantly improved the OS in patient groups of $<65$ and $\geq 65$ years compared with the control groups $(<65$ years: $\mathrm{HR}=0.76 ; 95 \% \mathrm{CI}=0.70-0.84 ; \mathrm{p}<0.001 ; \geq 65$ years: $\mathrm{HR}=0.79 ; 95 \% \mathrm{CI}=0.73-0.86 ; \mathrm{p}<0.001$; Figure 2). Subgroup analysis of the cancer type revealed a OS improvement of ICI immunotherapies in patients with non-small-cell lung cancer $(<65$ years: $\mathrm{HR}=0.71 ; 95 \%$ $\mathrm{CI}=0.59-0.85 ; \mathrm{p}<0.001 ; \geq 65$ years: $\mathrm{HR}=0.81 ; 95 \% \mathrm{CI}=0.70-0.94 ; \mathrm{p}=0.005)$, melanoma $(<65$ years: $\mathrm{HR}=0.76 ; 95 \% \mathrm{CI}=0.64-0.92 ; \mathrm{p}=0.004 ; \geq 65$ years: $\mathrm{HR}=0.66 ; 95 \% \mathrm{CI}=0.52-0.84 ; \mathrm{p}=0.001)$ and renal-cell carcinoma $(<65$ years: $\mathrm{HR}=0.65 ; 95 \% \mathrm{CI}=0.44-0.94 ; \mathrm{p}=0.024 ; \geq 65$ years: $\mathrm{HR}=0.80 ; 95 \% \mathrm{CI}=0.64-$ $1.01 ; \mathrm{p}=0.055)$, but not in patients with small-cell lung cancer, gastric cancer/gastro-esophageal junction cancer and head-and-neck squamous cell carcinoma (Table 2). Subgroup analysis based on ICI drug also indicated that anti-PD-1/PD-L1 inhibitors $(<65$ years: $\mathrm{HR}=0.76 ; 95 \% \mathrm{CI}=0.68-0.86 ; \mathrm{p}<0.001 ; \geq 65$ years: $\mathrm{HR}=0.72$; $95 \% \mathrm{CI}=0.65-0.81 ; \mathrm{p}<0.001)$, anti-PD-1 inhibitors $(<65$ years: $\mathrm{HR}=0.73 ; 95 \% \mathrm{CI}=0.63-0.84 ; \mathrm{p}<0.001$; $\geq 65$ years: $\mathrm{HR}=0.71 ; 95 \% \mathrm{CI}=0.63-0.81 ; \mathrm{p}<0.001)$ and $\mathrm{ICI}$ plus chemotherapy $(<65$ years: $\mathrm{HR}=0.72 ; 95 \%$ $\mathrm{CI}=0.58-0.88 ; \mathrm{p}=0.001 ; \geq 65$ years: $\mathrm{HR}=0.79 ; 95 \% \mathrm{CI}=0.66-0.94 ; \mathrm{p}=0.008)$ could improve OS in patient groups of $<65$ and $\geq 65$ years. Stratifying by therapy line of 1 and $\geq 2$, our results also indicated an improved OS in both patients aged $<65$ and $\geq 65$ years (Table 2 ).

Furthermore, our results found no significant differences in the OS improvement of ICI immunotherapies between patients aged $<65$ and $\geq 65$ years in comparison with the controls for each age group ( $p$ for interaction $=0.656$ ). The pooled within-trial interaction HRs confirmed that patients aged $<65$ and $\geq 65$ years were associated with a comparable benefit from ICI immunotherapies ( $\mathrm{HR}=1.01 ; 95 \% \mathrm{CI}=0.91-1.12 ; \mathrm{p}=0.796$; Supplementary Figure 1). Moreover, the pooled analysis of within-trial interaction HRs based on cancer type (Supplementary Figure 1), ICI drug and therapy line also obtained similar results.

\section{Overall survival \& cut-off age of 75 years}

Our results indicated that ICI immunotherapies significantly improved the OS in patients aged $<75$ years $(\mathrm{HR}=0.78 ; 95 \% \mathrm{CI}=0.68-0.89 ; \mathrm{p}<0.001$; Figure 3$)$, but patients aged $\geq 75$ years experienced less $\mathrm{OS}$ benefit from ICI immunotherapies $(\mathrm{HR}=0.88 ; 95 \% \mathrm{CI}=0.67-1.16 ; \mathrm{p}=0.377$; Figure 3 ). Moreover, after stratifying by cancer type (non-small-cell lung cancer and renal-cell carcinoma), therapy line (line 1 and line $\geq 2$ ) and ICI drug (anti-PD-1/PD-L1 inhibitors and anti-PD-1 inhibitors), the subgroup analyses yielded similar results (Table 2), indicating differences in the OS improvement of ICI immunotherapies between patients aged $<75$ and $\geq 75$ years compared with the controls for each age group. However, we observed that the number of patients in groups of $\geq 75$ years was limited and several in-depth subgroup analyses could not be performed owing to limited number of eligible studies.

\section{Progression-free survival \& cut-off age of 65 years}

Our results indicated that patients aged $<65$ years treated with ICI immunotherapies had a favorable PFS compared with the control groups $(\mathrm{HR}=0.69 ; 95 \% \mathrm{CI}=0.58-0.82 ; \mathrm{p}<0.001$; Figure 4). Similarly, for patients aged $\geq 65$ years, ICI immunotherapies could significantly improve the PFS compared with the control groups $(\mathrm{HR}=0.77$; $95 \% \mathrm{CI}=0.67-0.88 ; \mathrm{p}<0.001$; Figure 4). We observed similar results in the subgroup analyses stratified by non-small-cell lung cancer, melanoma, anti-PD-1/PD-L1 inhibitors, ICI plus chemotherapy, anti-PD-1 inhibitors and first line therapy; however, the ICI immunotherapies did not improve the PFS when ICI immunotherapies were treated as a second line therapy (Table 2).

Moreover, there was no differences in the PFS between patients aged $<65$ and $\geq 65$ years when the ICI immunotherapies were compared with the control group for each age group ( $\mathrm{p}$ for interaction $=0.369$ ), confirmed by the pooled within-trial interaction HRs ( $\mathrm{HR}=1.12 ; 95 \% \mathrm{CI}=0.98-1.28 ; \mathrm{p}=0.106$; Supplementary Figure 2). Similar results were obtained when we performed subgroup analysis based on cancer type (Supplementary Figure 2), ICI drug and therapy line. 


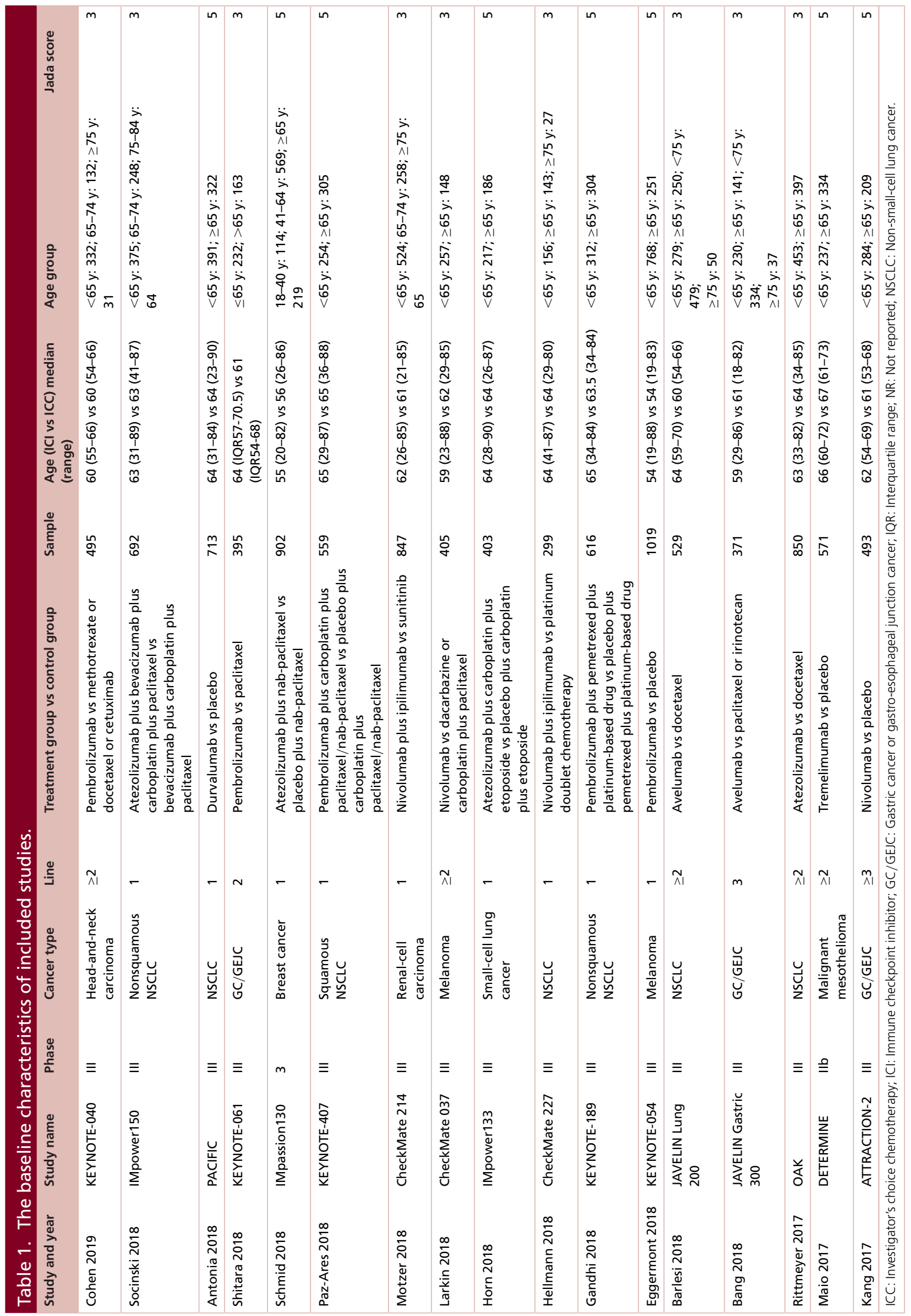




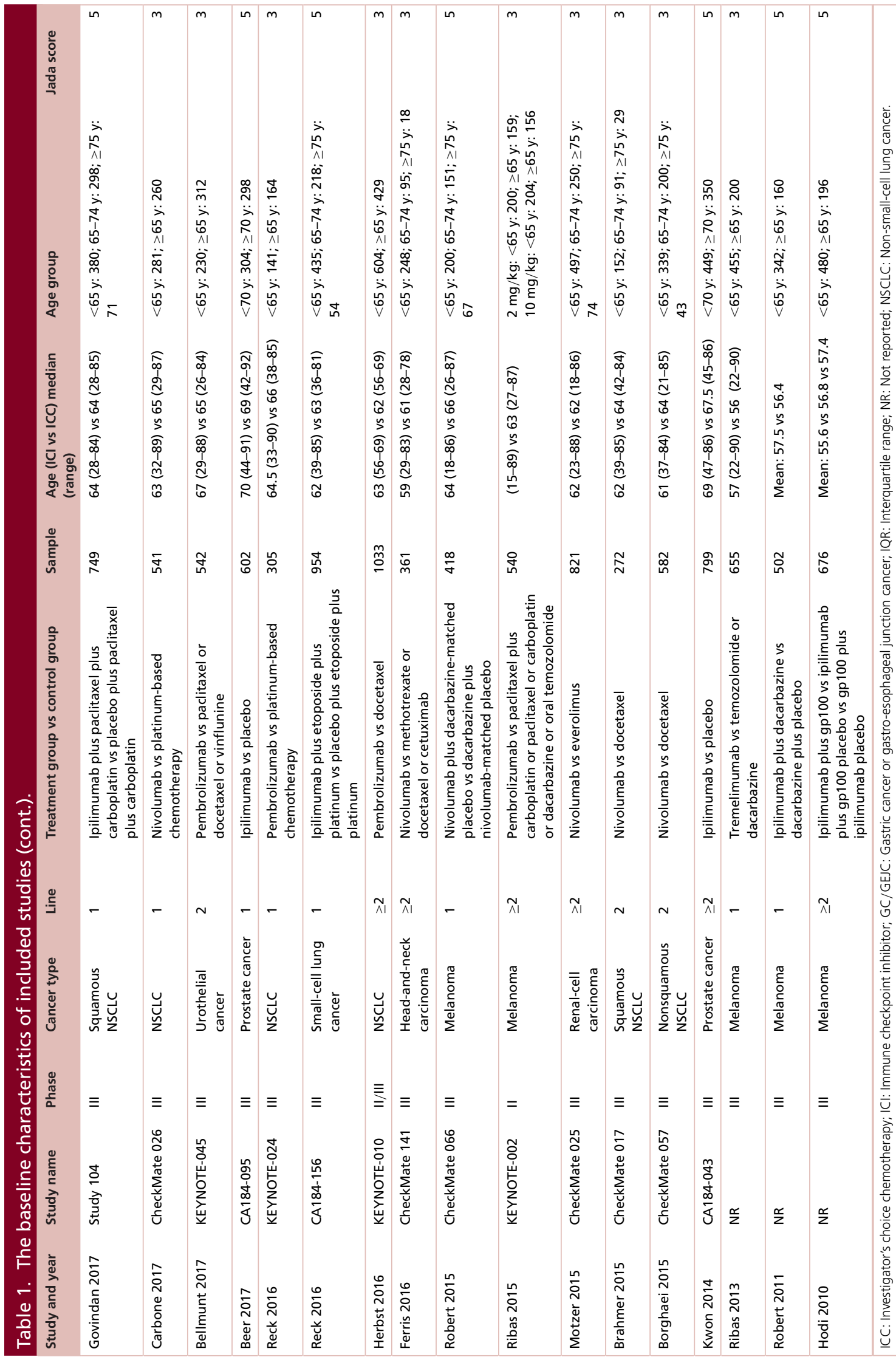




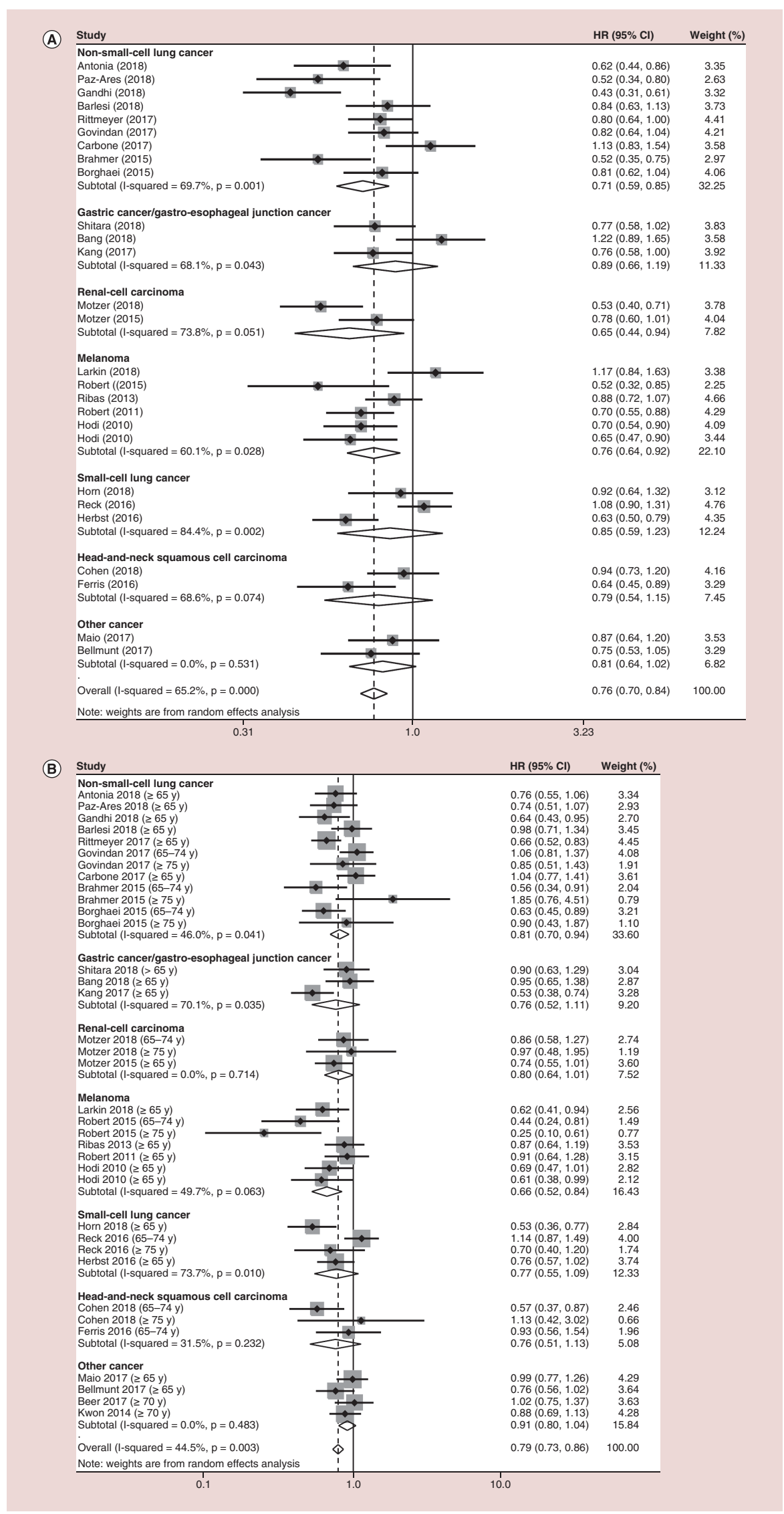

Figure 2. Hazard ratio of overall survival for patients aged $<65$ and $\geq 65$ years treated with immune checkpoint inhibitors when compared with those in control group. (A) Overall survival (OS) for patients aged $<65$ years; (B) OS for patients aged $\geq 65$ years. 
Systematic Review Huang, Gao, Song et al.

Table 2. The results of subgroup analyses for efficacy of immune checkpoint inhibitors in different age groups.

HR

Overall survival

$\begin{array}{ll}<65 \text { years } & 0.7 \\ \geq 65 \text { years } & 0.79 \\ <75 \text { years } & 0.78 \\ \geq 75 \text { years } & 0.88\end{array}$

Cancer type

NSCLC

\begin{tabular}{|c|c|c|c|}
\hline$<65$ years & 0.71 & $0.59-0.85$ & $<0.001$ \\
\hline$\geq 65$ years & 0.81 & $0.70-0.94$ & 0.005 \\
\hline$<75$ years & 0.77 & $0.65-0.91$ & 0.003 \\
\hline$\geq 75$ years & 1.03 & $0.73-1.44$ & 0.88 \\
\hline \multicolumn{4}{|l|}{ Melanoma } \\
\hline$<65$ years & 0.76 & $0.64-0.92$ & 0.004 \\
\hline$\geq 65$ years & 0.66 & $0.52-0.84$ & 0.001 \\
\hline \multicolumn{4}{|l|}{ SCLC } \\
\hline$<65$ years & 0.85 & $0.59-1.23$ & 0.400 \\
\hline$\geq 65$ years & 0.77 & $0.55-1.09$ & 0.136 \\
\hline \multicolumn{4}{|l|}{ GC/GEJC } \\
\hline$<65$ years & 0.89 & $0.66-1.19$ & 0.425 \\
\hline$\geq 65$ years & 0.76 & $0.52-1.11$ & 0.157 \\
\hline \multicolumn{4}{|l|}{$\mathrm{RCC}$} \\
\hline$<65$ years & 0.65 & $0.44-0.94$ & 0.024 \\
\hline$\geq 65$ years & 0.80 & $0.64-1.01$ & 0.055 \\
\hline$<75$ years & 0.68 & $0.55-0.85$ & $<0.001$ \\
\hline$\geq 75$ years & 1.11 & $0.69-1.77$ & 0.670 \\
\hline \multicolumn{4}{|l|}{ HNSCC } \\
\hline$<65$ years & 0.79 & $0.54-1.15$ & 0.218 \\
\hline$\geq 65$ years & 0.76 & $0.51-1.13$ & 0.049 \\
\hline
\end{tabular}

Therapy line

Line 1

\begin{tabular}{llll}
$<65$ years & 0.72 & $0.60-0.87$ & 0.001 \\
\hline$\geq 65$ years & 0.82 & $0.71-0.94$ & 0.005 \\
\hline 75 years & 0.80 & $0.64-1.01$ & 0.059 \\
\hline$\geq 75$ years & 0.67 & $0.42-1.07$ & 0.095 \\
\hline
\end{tabular}

Line $\geq 2$

\begin{tabular}{|llll}
$<65$ years & 0.79 & $0.71-0.87$ & $<0.001$ \\
\hline$\geq 65$ years & 0.76 & $0.69-0.84$ & $<0.001$ \\
\hline$<75$ years & 0.76 & $0.65-0.89$ & 0.001 \\
\hline$\geq 75$ years & 1.11 & $0.81-1.53$ & 0.521 \\
\hline
\end{tabular}

ICI drug

PD-1/PD-L1

\begin{tabular}{|c|c|c|c|}
\hline$<65$ years & 0.76 & $0.68-0.86$ & $<0.001$ \\
\hline$\geq 65$ years & 0.72 & $0.65-0.81$ & $<0.001$ \\
\hline$<75$ years & 0.72 & $0.62-0.84$ & $<0.001$ \\
\hline$\geq 75$ years & 0.92 & $0.60-1.41$ & 0.7 \\
\hline
\end{tabular}


Table 2. The results of subgroup analyses for efficacy of immune checkpoint inhibitors in different age groups (cont.).

\section{HR}

\section{PD-1}

\begin{tabular}{|c|c|}
\hline$<65$ years & 0 . \\
\hline$\geq 65$ years & 0 \\
\hline$<75$ years & 0. \\
\hline$\geq 75$ years & 0 \\
\hline
\end{tabular}

\section{CTLA-4}

$<65$ years

$\geq 65$ years

0.73

0.91

0.91

0.82

0.93

ICI + ICC

\begin{tabular}{|l|l|}
\hline$<65$ years \\
\hline$>65$ years \\
\hline
\end{tabular}

0.72

0.79

Progression-free survival

Overall

\begin{tabular}{|lc}
\hline$<65$ years & 0.69 \\
\hline$\geq 65$ years & 0.77 \\
\hline$<75$ years & 0.77 \\
\hline$\geq 75$ years & 0.85 \\
\hline
\end{tabular}

\section{Cancer type}

NSCLC

\begin{tabular}{|c|c|c|c|}
\hline$<65$ years & 0.64 & $0.51-0.80$ & $<0.001$ \\
\hline$\geq 65$ years & 0.76 & $0.63-0.92$ & 0.004 \\
\hline$<75$ years & 0.70 & $0.57-0.85$ & $<0.001$ \\
\hline$\geq 75$ years & 0.91 & $0.64-1.30$ & 0.597 \\
\hline \multicolumn{4}{|l|}{ Melanoma } \\
\hline$<65$ years & 0.48 & $0.40-0.59$ & $<0.001$ \\
\hline$\geq 65$ years & 0.63 & $0.49-0.80$ & $<0.001$ \\
\hline \multicolumn{4}{|l|}{ SCLC } \\
\hline$<65$ years & 0.81 & $0.69-0.96$ & 0.012 \\
\hline$\geq 65$ years & 0.86 & $0.71-1.04$ & 0.119 \\
\hline \multicolumn{4}{|c|}{ Therapy line } \\
\hline \multicolumn{4}{|l|}{ Line 1} \\
\hline$<65$ years & 0.63 & $0.52-0.76$ & $<0.001$ \\
\hline$\geq 65$ years & 0.69 & $0.59-0.81$ & $<0.001$ \\
\hline$<75$ years & 0.57 & $0.49-0.67$ & $<0.001$ \\
\hline$\geq 75$ years & 0.69 & $0.42-1.13$ & 0.142 \\
\hline \multicolumn{4}{|l|}{ Line $\geq 2$} \\
\hline$<65$ years & 0.78 & $0.56-1.10$ & 0.157 \\
\hline$\geq 65$ years & 0.89 & $0.72-1.09$ & 0.264 \\
\hline$<75$ years & 0.92 & $0.65-1.28$ & 0.61 \\
\hline$\geq 75$ years & 0.98 & $0.63-1.51$ & 0.921 \\
\hline \multicolumn{4}{|l|}{$\mathrm{ICl}$ drug } \\
\hline \multicolumn{4}{|l|}{ PD-1/PD-L1 } \\
\hline$<65$ years & 0.70 & $0.58-0.84$ & $<0.001$ \\
\hline$\geq 65$ years & 0.77 & $0.67-0.89$ & $<0.001$ \\
\hline$<75$ years & 0.82 & $0.60-1.10$ & 0.183 \\
\hline$\geq 75$ years & 0.91 & $0.66-1.25$ & 0.402 \\
\hline
\end{tabular}

CTLA-4: Cytotoxic T-lymphocyte-associated antigen 4; GC/GEJC: Gastric cancer/gastro-esophageal junction cancer; HNSCC: Head-and-neck squamous cell carcinoma; HR: Hazard ratio; 12: Degree of heterogeneity; ICC: Investigator's choice chemotherapy; ICI: Immune checkpoint inhibitor; NSCLC: Non-small-cell lung cancer; p: p for the HR; PD-1: Programmed cell death protein-1; PD-L1: Programmed cell death-ligand 1; RCC: Renal-cell carcinoma; SCLC: Small-cell lung cancer.

$p$ for $H R$

$\begin{array}{lc}0.63-0.84 & <0.001 \\ 0.63-0.81 & <0.001 \\ 0.63-0.78 & <0.001 \\ 0.49-1.68 & 0.761\end{array}$

$\begin{array}{ll}0.71-0.94 & 0.005 \\ 0.84-1.02 & 0.129\end{array}$

$\begin{array}{ll}0.58-0.88 & 0.001\end{array}$

$\begin{array}{ll}0.66-0.94 & 0.008\end{array}$

$\begin{array}{llc}0.69 & 0.58-0.82 & <0.001 \\ 0.77 & 0.67-0.88 & <0.001 \\ 0.77 & 0.58-1.03 & 0.081 \\ 0.85 & 0.61-1.19 & 0.354\end{array}$

0.354 
Table 2. The results of subgroup analyses for efficacy of immune checkpoint inhibitors in different age groups (cont.). HR

$95 \% \mathrm{Cl}$

p for HR

PD-1

\begin{tabular}{llll}
$<65$ years & 0.6 & $0.48-0.75$ & $<0.001$ \\
$\geq 65$ years & 0.75 & $0.64-0.89$ & 0.001 \\
$<75$ years & 0.75 & $0.58-0.97$ & 0.029 \\
$\geq 75$ years & 1.24 & $0.73-2.10$ & 0.431 \\
\hline $\mathrm{ICl}+\mathrm{ICC}$ & & & $<0.001$ \\
\hline$<65$ years & 0.64 & $0.52-0.81$ & $0.59-0.77$
\end{tabular}

CTLA-4: Cytotoxic T-lymphocyte-associated antigen 4; GC/GEJC: Gastric cancer/gastro-esophageal junction cancer; HNSCC: Head-and-neck squamous cell carcinoma; HR: Hazard ratio; 12: Degree of heterogeneity; ICC: Investigator's choice chemotherapy; ICl: Immune checkpoint inhibitor; NSCLC: Non-small-cell lung cancer; p: p for the HR; PD-1: Programmed cell death protein-1; PD-L1: Programmed cell death-ligand 1; RCC: Renal-cell carcinoma; SCLC: Small-cell lung cancer.

(A)

Study

Non-small-cell lung cancer

Govindan 2017 (<65 y)

Govindan 2017 (65-74 y)

Brahmer $2015(<65 \mathrm{y})$
Brahmer $2015(65-74 \mathrm{y})$

Borghaei $2015(<65 y)$

Borghaei $2015(65-74$ y)

Subtotal (1-squared 5 y

Renal-cell carcinoma

Motzer $2018(<65 \mathrm{y})$

Motzer $2018(65-74 \mathrm{y})$

Motzer 2015 (<65 y)

Subtotal (l-squared $=45.0 \%, p=0.141)$

Other cancer

Cohen 2018 (<65 y)

Cohen $2018(65-74 y)$

Reck $2016(<65 y)$

Reck $2016(65-74$ y)

Reck $2016(65-74 \mathrm{y})$
Robert $2015(<65 \mathrm{y})$

Robert $2015(<65 y)$

Subtotal (I-squared $=74.3 \%, p=0.00$

Note: weights are from random effects analysis

$$
0.24
$$

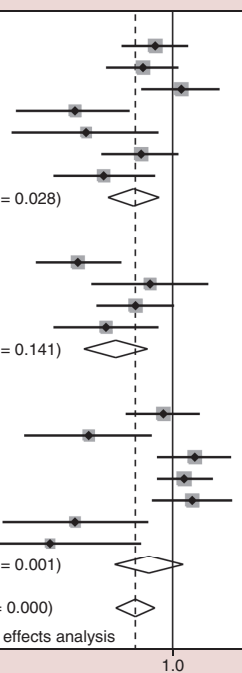

HR (95\% Cl) Weight (\%)

$0.89(0.71,1.11) \quad 6.76$ $0.82(0.64,1.04) \quad 6.53$ $0.82(0.64,1.04) \quad 6.53$ $0.52(0.35,0.75) \quad 4.92$ $0.56(0.34,0.91) \quad 3.92$ $0.81(0.62,1.04) \quad 6.34$ $0.81(0.62,1.04) \quad 6.34$

$\begin{array}{lr}0.63(0.45,0.89) & 5.36 \\ 0.77(0.65,0.91) & 40.04\end{array}$

$0.53(0.40,0.71) \quad 6.00$ $0.86(0.58,1.27) \quad 4.80$ $0.88(0.60,1.01) \quad 6.32$

$0.64(0.45,0.91) \quad 5.23$

$0.64(0.45,0.01) \quad 5.23$

$0.94(0.73,1.20) \quad 6.46$

$0.94(0.73,1.20) \quad 6.46$

$1.16(0.90,1.48) \quad 6.46$

$1.08(0.90,1.31) \quad 7.18$

$1.14(0.87,1.49) \quad 6.21$

$\begin{array}{ll}1.14(0.87,1.49) & 6.21 \\ 0.52(0.32,0.85) & 3.88\end{array}$

$0.44(0.24,0.81) \quad 3.89$

$0.44(0.24,0.81) \quad 2.99$

$0.78(0.68,0.89) \quad 100.00$

4.17

(C)

\section{Study}

Non-small-cell lung cancer

Socinski $2018(<65 \mathrm{y})$

Socinski 2018 (65-74 y)

Hellmann $2018(<65 \mathrm{y})$

Barlesi $2018(<75 y)$

Brahmer 2015 (<65 y)

Brahmer 2015 (65-74 y)

Borghaei 2015 (<65y)

Borghaei 2015 (65-74 y)

Subtotal $($ - -squared $=74.2 \%, p=0.000)$

Other cancer

Bang 2018 (< 75 y)

Subtotal (I-squared $=. \%, p=$.)

Overall (I-squared $=89.2 \%, p=0.000)$

Note: weights are from random effects analysis

0.32

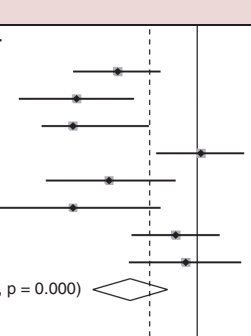

HR $(95 \% \mathrm{Cl}) \quad$ Weight (\%)

$0.65(0.51,0.82) \quad 11.67$

$0.52(0.38,0.71) \quad 11.03$

$0.51(0.43,0.77) \quad 11.22$

$1.02(0.80,1.29) \quad 11.65$

$0.62(0.44,0.89) \quad 10.66$

$0.51(0.32,0.82) \quad 9.49$

$0.89(0.70,1.13) \quad 11.65$

$0.94(0.69,1.27) \quad 11.10$

$0.70(0.57,0.85) \quad 88.48$

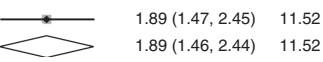

$0.77(0.58,1.03) \quad 100.00$
Barlesi $2018(<75 \mathrm{y})$

Overall (I-squared $=70.5 \%, p=0.000)$

(B)

Study

Non-small-cell lung cancer

Barlesi 2018 ( $\geq 75$ y)

Govindan 2017 ( $\geq 75 \mathrm{y})$

Brahmer 2015 ( $\geq 75 \mathrm{y})$

Borghaei 2015 ( $\geq 75$ y)

Subtotal (l-squared $=0.0 \%, p=0.490)$

Renal-cell carcinoma

Motzer 2018 ( $\geq 75 \mathrm{y}$ )

Motzer $2015(\geq 75 \mathrm{y})$

Subtotal $($ - -squared $=0.0 \%, p=0.620)$

Other cancer

Cohen $2018(\geq 75 y)$

Bang 2018 ( $\geq 75 \mathrm{y})$

Reck 2016 ( $\geq 75$ y)

Robert 2015 ( $\geq 75$ y)

Subtotal (1-squared $=46.4 \%, p=0.133)$

Overall (l-squared $=30.5 \%, p=0.165)$

Note: weights are from random effects analysis

0.1

HR (95\% Cl) Weight (\%)

$1.16(0.54,2.47) \quad 9.32$ $0.85(0.51,1.43) \quad 15.29$ $1.85(0.76,4.51) \quad 7.35$ $0.90(0.43,1.87) \quad 9.78$

$1.03(0.73,1.44) \quad 41.74$

$0.97(0.48,1.95) \quad 10.46$ $1.23(0.66,2.31) \quad 12.14$ $1.11(0.69,1.77) \quad 22.59$

$13(0.42,3.02) \quad 6.24$ $0.72(0.31,1.67) \quad 8.01$ $0.70(0.40,1.20) \quad 14.24$ $0.25(0.10,0.61) \quad 7.17$ $0.62(0.36,1.07) \quad 35.66$

$0.88(0.67,1.16) 100.00$

(D)

Study Non-small-cell lung cancer Socinski 2018 (75-84 y) Hellmann $2018(\geq 75 \mathrm{y})$ Barlesi $2018(\geq 75 y)$ Brahmer $2015(\geq 75 \mathrm{y})$ Borghaei 2015 ( $\geq 75 \mathrm{y}$ )

Subtotal $(\mathrm{I}-$ squared $=13.2 \%, p=0.330$ )

Other cancer

Bang 2018 ( $\geq 75$ y)

Subtotal (I-squared $=. \%, \mathrm{p}=$.)

Overall (I-squared $=12.7 \%, p=0.334$ )

Note: weights are from random effects analysis

0.14

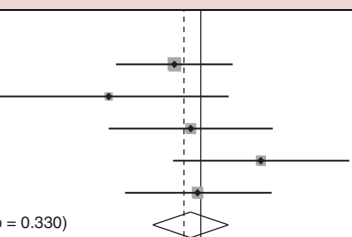

HR (95\% Cl) Weight (\%)

$0.78(0.45,1.35) \quad 28.97$ $0.42(0.14,1.30) \quad 8.48$ $0.91(0.42,1.97) \quad 16.46$ $1.76(0.77,4.05) \quad 14.51$ $0.97(0.49,1.95) \quad 19.97$ $0.91(0.64,1.30) \quad 88.39$

$0.53(0.20,1.31) \quad 11.61$ $0.53(0.21,1.36) \quad 11.61$ $0.85(0.61,1.19) \quad 100.00$ 7.14

Figure 3. Hazard ratio of overall survival and progression-free survival for patients aged $<75$ and $\geq 75$ years treated with immune checkpoint inhibitors when compared with those in control group. (A) Overall survival (OS) for patients aged $<75$ years; (B) OS for patients aged $\geq 75$ years; (C) progression-free survival (PFS) for patients aged $<75$ years; (D) PFS for patients aged $\geq 75$ years. 
(A) Study

HR (95\% Cl) Weight (\%)

Non-small-cell lung cancer

Socinski

Antonia

Paz-Ares

Hellmann

Gandhi

Barlesi

Carbone

Reck

Brahmer

Borghaei

Subtotal (I-squared $=82.3 \%, p=0.000$

Melanoma

Eggermont

Ribas

Ribas

Subtotal (I-squared $=0.0 \%, p=0.444)$

Small-cell lung cancer

Horn

Herbst

Subtotal (I-squared $=0.0 \%, p=0.571)$

Other cancer

Schmid

Schmid

Bang

Subtotal (I-squared $=91.2 \%, p=0.000)$

Overall (I-squared $=85.6 \%, p=0.000)$

Note: weights are from random effects analysis

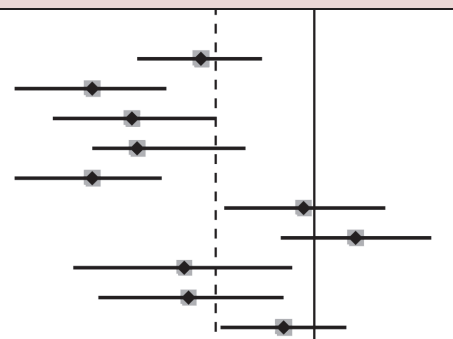

$0.65(0.51,0.82) \quad 5.92$

$0.43(0.32,0.57) \quad 5.63$

$0.50(0.37,0.69) \quad 5.49$

$0.51(0.43,0.77) \quad 5.61$

$0.43(0.32,0.56) \quad 5.68$

$0.96(0.71,1.31) \quad 5.53$

$1.17(0.88,1.56) \quad 5.64$

$0.61(0.40,0.92) \quad 4.84$

$0.62(0.44,0.89) \quad 5.24$

$0.89(0.70,1.13) \quad 5.91$

$0.64(0.51,0.80) \quad 55.50$

$0.57(0.41,0.80) \quad 5.36$

$0.47(0.34,0.66) \quad 5.37$

$0.42(0.30,0.59) \quad 5.33$

$0.48(0.40,0.59) \quad 16.06$

$0.76(0.57,1.01) \quad 5.64$

$0.84(0.69,1.02) \quad 6.13$

$0.81(0.69,0.96) \quad 11.77$

$0.79(0.53,1.16) \quad 5.00$

$0.84(0.70,1.01) \quad 6.18$

$1.96(1.43,2.67) \quad 5.49$

$1.09(0.62,1.91) \quad 16.67$

$0.69(0.58,0.82) \quad 100.00$

0.3

1.0

3.33

(B) Study

HR ( $95 \% \mathrm{Cl})$

Weight (\%)

Non-small-cell lung cancer

Socinski 2018 (65-74 y)

Socinski 2018 (75-84 y)

Antonia 2018 ( $\geq 65$ y)

Paz-Ares 2018 ( $\geq 65$ y)

Hellmann 2018 ( $\geq 65$ y)

Gandhi 2018 ( $\geq 65$ y)

Barlesi 2018 ( $\geq 65 y$ )

Carbone 2017 ( $\geq 65 \mathrm{y})$

Reck 2016 ( $\geq 65$ y)

Brahmer 2015 (65-74 y)

Brahmer 2015 ( $\geq 75$ y)

Borghaei 2015 (65-74 y)

Borghaei 2015 ( $\geq 75$ y)

Subtotal $($ I-squared $=67.1 \%, p=0.000)$

Melanoma

Eggermont 2018 ( $\geq 65 \mathrm{y}$ )

Ribas 2015 ( $\geq 65$ y)

Ribas 2015 ( $\geq 65$ y)

Subtotal $(\mathrm{I}$-squared $=0.0 \%, p=0.734)$

Small-cell lung cancer

Horn 2018 ( $\geq 65$ y)

Herbst 2016 ( $\geq 65 \mathrm{y}$ )

Subtotal $(\mathrm{I}$-squared $=0.4 \%, p=0.316)$

Other cance

Schmid 2018 ( $\geq 65$ y)

Bang 2018 ( $\geq 65 \mathrm{y}$ )

Subtotal $(\mathrm{I}$-squared $=86.8 \%, \mathrm{p}=0.006)$

Overall (I-squared $=62.9 \%, p=0.000$ )

Note: weights are from random effects analysis

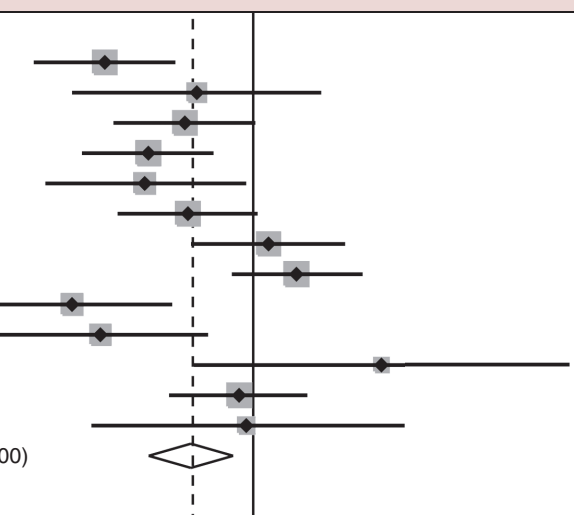

$0.52(0.38,0.71) \quad 5.84$

$0.78(0.45,1.35) \quad 3.55$

$0.74(0.54,1.01) \quad 5.84$

$0.63(0.47,0.84) \quad 6.10$

$0.62(0.40,0.97)-4.46$

$0.75(0.55,1.02)$

$1.07(0.76,1.50) \quad 5.53$

$1.21(0.91,1.62) \quad 6.13$

$0.45(0.29,0.70) \quad 4.48$

$0.51(0.32,0.82) \quad 4.20$

$76(0.77,4.05) \quad 2.04$

$0.94(0.69,1.27) \quad 5.93$

$0.97(0.49,1.95) \quad 2.66$

$0.76(0.63,0.92) \quad 62.64$

$0.55(0.32,0.93) \quad 3.67$

$0.70(0.48,1.01) \quad 5.18$

$0.60(0.41,0.88) \quad 5.07$

$0.63(0.49,0.80) \quad 13.92$

$0.76(0.56,1.03) \quad 5.94$

$0.93(0.72,1.19) \quad 6.56$

$0.86(0.71,1.04) \quad 12.50$

$0.69(0.51,0.94) \quad 5.92$

$1.38(0.94,2.04) \quad 5.01$

$0.97(0.49,1.90) \quad 10.94$

$0.77(0.67,0.88) \quad 100.00$

0.247

1.0

Figure 4. Hazard ratio of progression-freesurvival for patients aged $<65$ and $\geq 65$ years treated with immune checkpoint inhibitors when compared with those in control group. (A) Progression-free survival (PFS) for patients aged $<65$ years; (B) PFS for patients aged $\geq 65$ years. 
Progression-free survival \& cut-off age of 75 years

ICI immunotherapies tended toward a favorable PFS in patients aged $<75$ years, although statistical significance was not reached $(\mathrm{HR}=0.77 ; 95 \% \mathrm{CI}=0.58-1.03$; Figure 3 ); however, we observed less PFS benefit form ICI immunotherapies in patients aged $\geq 75$ years $(\mathrm{HR}=0.85 ; 95 \% \mathrm{CI}=0.61-1.19 ; \mathrm{p}=0.354$; Figure 3$)$. Similar results were observed in the subgroup analysis of non-small-cell lung cancer and first line therapy. ICI immunotherapies treated as a second line therapy did not significantly improve the PFS in patients aged both $<75$ and $\geq 75$ years compared with the control groups.

\section{Discussion}

ICI immunotherapies have changed the landscape of cancer treatment for several advanced solid tumors [52]. New cancer cases and deaths due to cancer which primarily occur in older patients aged $\geq 65$ years [4]. However, the evidence regarding the efficacy of ICI immunotherapies in older adults with cancer are limited because such older patients continue to be under-represented in cancer clinical trials considering the safety profile of ICI drugs. ICI immunotherapies can re-activate host immune function and subsequently enhance the antitumor response through inhibiting the PD-1 or CTLA-4 pathways; therefore, host immune function is critically important for the efficacy of ICI drugs [53,54]. Theoretically, the age-related immunosenescence of older patients may negatively impact the clinical efficacy of ICI immunotherapies; however, no studies have drawn definitive conclusions specific to older patients.

We included 34 Phase II or III studies containing a total of 20,511 patients to evaluate the clinical efficacy of ICI immunotherapies among different age groups ( $<65$ vs $\geq 65$ years and $<75$ vs $\geq 75$ years). Our results indicated that ICI immunotherapies could prolong the OS and PFS in the patient groups of $<65$ and $\geq 65$ years compared with the control groups, which was not significantly different when compared with the controls for each age group. In addition, our results indicated that ICI immunotherapies improved the OS and PFS in patients aged $<75$ years, while patients aged $\geq 75$ years experienced less survival benefit form ICI immunotherapies. However, there may not be enough power to definitively make a conclusion about the effectiveness of ICI immunotherapies in patients aged $\geq 75$ years due to limited patients in groups of $\geq 75$ years ( $~ 3 \%, 630$ patients). Further large-scale studies are needed to explore the impact of age on ICI efficacy in cancer patients.

As a revolutionary cancer treatment, ICI immunotherapies combined with or without chemotherapy have become an alternative first-line or subsequent treatment for several cancers $[21,22,24,26,39,50]$. The pooled results indicated an improved OS and PFS for ICI immunotherapies in patients aged $<65$ and $\geq 65$ years; however, the magnitude of the benefit observed in the $\geq 65$ years group was slightly lower than that observed in the $<65$ year age group, although not statistically significant, particularly for non-small-cell lung cancer. We also observed less survival benefit form ICI immunotherapies in patients aged $\geq 75$ years, but the limited number of included patients may affect the reliability of our results. Currently, although it was unclear how the patients' age affected the antitumor response and clinical efficacy of ICI immunotherapies, several studies have reported that the number and functional diversity of $\mathrm{T}$ cells decreased with age due to thymic involution and the conversion of naive $\mathrm{T}$ cells into memory $\mathrm{T}$ cells $[8,55]$. Thus, it was hypothesized that immunosenescence, which mainly occurred with regards to $\mathrm{T}$ cell-mediated immunity, was an important contributing factor to the limited efficacy of ICI immunotherapies in older patients [6,7]. Surprisingly, however, we found that melanoma patients aged $\geq 65$ years could benefit more from immunotherapy than younger patients. The potential explanation was that age-related differences in intratumoral immune microenvironment were different in different tumor. Indeed, a melanoma clinical and animal study by Kugel et al. found that the likelihood of response to anti-PD-1 immunotherapies increased with age in melanoma patients and the melanoma animal models showed that older mice had a lower Tregs level and higher $\mathrm{CD}^{+}:$Treg ratio, confirmed by the human melanoma biopsies [56]. Thus, age-dependent ICI efficacy may be different between cancer diseases, which need further studies to explore the correlation. Among the eligible trials, there were ten that evaluated the efficacy of ICI immunotherapies among cancer patients aged $\geq 75$ years [22,28-30,35,40,43,45-47], nine of which consistently showed that ICI immunotherapies did not improve the OS in cancer patients aged $\geq 75$ years, regardless of the type of cancer and ICI drug. CheckMate 017 by Brahmer $e t$ al. assessed the efficacy of nivolumab versus chemotherapy in previously treated, advanced squamous-cell non-small-cell lung cancer; the results showed that nivolumab could prolong the $\mathrm{OS}$ in patients aged $<65(\mathrm{HR}=0.52 ; 95 \% \mathrm{CI}=0.35-0.75)$ and $65-74$ years $(\mathrm{HR}=0.56 ; 95 \% \mathrm{CI}=0.34-0.91)$, but not in patients aged $\geq 75$ years $(\mathrm{HR}=1.85 ; 95 \% \mathrm{CI}=0.74-4.51)[46]$. CheckMate 057 was a Phase III study comparing nivolumab with docetaxel in patients previously treated with advanced nonsquamous non-small-cell lung cancer, which reported that OS was longer in the nivolumab group 
compared with the chemotherapy group in the overall cohort. Moreover, the subgroup analysis based on age showed that the HR for the OS was $0.81(95 \% \mathrm{CI}=0.62-1.04)$ in patients aged $<65$ years, $0.63(95 \% \mathrm{CI}=0.45-0.89)$ in patients aged $65-74$ years and $0.90(95 \% \mathrm{CI}=0.43-1.87)$ in patients aged $\geq 75$ years [47]. Moreover, in CheckMate 025, the nivolumab group was associated with a favorable OS compared with everolimus in advanced renal-cell carcinoma patients aged $<65(\mathrm{HR}=0.78 ; 95 \% \mathrm{CI}=0.60-1.01)$ and $65-74$ years $(\mathrm{HR}=0.64 ; 95 \%$ $\mathrm{CI}=0.45-0.91)$; however, nivolumab did not prolong the $\mathrm{OS}$ in patients aged $\geq 75$ years $(\mathrm{HR}=1.23$; $95 \%$ $\mathrm{CI}=0.66-2.31)[45]$. Further studies are required to explore the underlying mechanism of the impact of age on the efficacy of ICI immunotherapies in cancer patients.

Most trials have assessed the impact of age on the efficacy of ICI immunotherapies using a numerical cut-off age of 65 years. An accurate definition of a cut-off age for 'older patients' remains controversial in clinical practice when considering the difference between chronological and physiological age. While the included studies used chronological age, the aging of the organs and functional status of an individual was a physiological process; thus, the chronological age could not comprehensively reflect the of physiological age status of the patients [57]. In general, 'older patients' represented a heterogeneous population and the incidence of comorbidities and poor performance status typically increased with age $[57,58]$. However, the older patients included in clinical trials were more likely to have a low number of comorbidities and good performance status when considering the drug safety profile, which did not represent the 'real world' situation of older patients. Therefore, the definition of 'older patients' in studies involving ICI immunotherapies should include an evaluation of the functional status, comorbidities, cognition, nutritional status, social support and psychological status of the patients [57]. Therefore, further studies are required to precisely define 'older patients' for ICI immunotherapies when considering the efficacy, safety and cost, as well as to evaluate the efficacy of ICI immunotherapies in "older patients".

Preclinical evidence has shown that chemotherapy could activate and enhance the T cell-mediated antitumor immune response via killing tumor cells, promote tumor-associated antigen release and stimulate dendritic cell maturation $[59,60]$. Indeed, emerging clinical trials have reported that treatment with immunotherapies in combination with chemotherapy displayed a synergistic antitumor effect and combination therapies could improve the prognosis of cancer patients $[18,20,21,26]$. However, it was unclear whether age has an effect on the efficacy between the use of combination therapies and ICI immunotherapies alone. Therefore, we further evaluated the impact of age on the efficacy of ICI immunotherapy in combination with chemotherapy and the results showed a similar result with the overall analysis. Further studies are required to explore which chemotherapeutic drug is suitable for being combined with ICI immunotherapies, as well as the optimum dose of the chemotherapeutic drug.

There were several limitations associated with the present study. First, the age cut-off ( 65 and 75 years) in this study was predefined by the included trials and we could not explore the optimal age cut-off for ICI efficacy in cancer patients. Meanwhile, the pre-defined age cut-off lead to limited patients aged $>75$ years (a total of 630 patients $[\sim 3 \%]$ ) and limited cancer diseases being included in our study. Thus, potential patients with more survival benefit from ICI such as melanoma were not included in the subgroup analysis of patient aged $\geq 75$ years, which may underestimate the ICI efficacy and affect the reliability of our results. Further large-scale multicenter studies are required to evaluate the efficacy of ICI immunotherapies in older patients and to explore the optimal age cut-off for ICI immunotherapies according different cancer diseases. Second, although our study have performed subgroup analysis based on cancer type, therapy line and ICI drug, we could not control for or avoid any potentially confounding factors and relevant bias (e.g., smoking status, comorbidities). For example, lung cancer patients and head and neck cancer patients are more likely to be current or former smokers and have comorbidities. Several studies reported that smokers were associated with higher ICI efficacy for lung cancer, but not for head and neck cancer $[61,62]$ and we could not assess comorbidities because patients with numerous comorbidities were frequently excluded from clinical studies. Furthermore, there may be an important difference in comorbidities between some patients groups. For example, chronic obstructive pulmonary disease and coronary artery disease are common smoker-related comorbidities in non-small-cell lung cancer patients and would be more problematic in patients aged $\geq 75$ years than in patients aged $\geq 65$ years, which may limit the potential ICI efficacy in that patient group. Oppositely, this situation does not apply to the older patients with melanoma because melanoma patients are much less likely to have smoker-related comorbidities. Thus, further studies are needed to determine whether and how these different confounding factors among various cancers affect ICI efficacy. Third, we could not assess the immune-related adverse events of ICI immunotherapies stratified by age and it was unclear as to whether the benefit of ICI immunotherapies in older patients (particularly in patients aged $\geq 65$ years) outweighed the associated adverse events. 


\section{Conclusion}

Our results indicate that ICI immunotherapies can prolong the OS and PFS in patient groups aged $<65$ and $\geq 65$ years without significant differences when compared with the controls for each age group. However, our results cannot definitively conclude that ICI immunotherapies are ineffective for patients aged $\geq 75$ years due to limited patients in this group. Further large-scale multicenter studies are required to explore the impact of age on ICI efficacy and the optimal age cut-off for ICI immunotherapies according different cancer diseases.

\section{Future perspective}

Immunotherapies based on ICI have become an attractive treatment option and changed the therapeutic landscape for several advanced malignancies. However, older adults are under-represented in clinical studies evaluating ICI immunotherapies and age-related immunosenescence may negatively impact the efficacy of ICI. We included two or three randomized controlled trials to evaluate the clinical efficacy of ICI immunotherapies among different age groups. Our results indicate that ICI immunotherapies can prolong the OS and PFS in patient groups aged $<65$ and $\geq 65$ years without significant differences. Moreover, ICI can improve the survival in patients aged $<75$ years, but less survival benefit were observed in patients aged $\geq 75$ years. However, there may not be enough power to definitively make a conclusion about the effectiveness of ICI immunotherapies in patients aged $\geq 75$ years due to limited patients in groups. Further studies are required to explore the underlying mechanism of the impact of age on the efficacy of ICI immunotherapies and to precisely define 'older patients' for ICI immunotherapies when considering the efficacy, safety and cost, as well as to evaluate the efficacy of ICI immunotherapies in 'older patients'.

\section{Summary points}

\section{Background}

- Immunotherapies based on immune checkpoint inhibitors (ICI) have become an attractive treatment option and changed the therapeutic landscape for several advanced malignancies.

- Older adults comprise the majority of patients diagnosed with cancer in the 'real world', but older adults are under-represented in clinical studies evaluating $\mathrm{ICl}$ immunotherapies.

- The age-related immunosenescence may negatively impact the efficacy of ICl.

- It is inappropriate to manage older cancer patients simply using the clinical evidence extrapolated from studies of younger cancer patients.

Materials \& methods

- PubMed, Embase and conference databases were systematically searched for studies that reported the efficacy of $\mathrm{ICl}$ according to patient age.

- The inclusion criteria consisted of eligible patients diagnosed with solid cancer, ICI immunotherapies as interventions and overall survival (OS) and progression-free survival (PFS) as outcomes.

- Subgroup, metaregression analysis and within-trial interaction hazard ratio were used to assess the impact of age on the efficacy of ICl.

Results

- A total of 34 studies containing 20,511 cancer patients were included. ICI could improve the OS and PFS in both patient groups of $<65$ and $\geq 65$ years compared with the control groups, without significant differences when compared with the controls for each age group.

- ICl immunotherapies improved the OS and PFS in patients aged $<75$ years, while patients aged $\geq 75$ years experienced less survival benefit form ICI immunotherapies when the cut-off age was set to 75 years.

- The age-related immunosenescence could negatively impact the efficacy of ICl.

Conclusion

- ICI has comparable efficacy in cancer patients aged $<65$ and $\geq 65$ years. Cancer patients aged $\geq 75$ years need more attention in the future clinical trials.

- Further studies are required to explore the underlying mechanism of the impact of age on the efficacy of ICI immunotherapies. 


\section{Author contributions}

X Huang, P Gao and Z Wang were responsible for conception and design of the study. All authors contributed to the acquisition, analysis, interpretation of data, manuscript drafting and revising, final approval of the version to be submitted and published and agreement to be accountable for all aspects of the work in ensuring that questions related to the accuracy or integrity of any part of the work are appropriately investigated and resolved.

\section{Acknowledgments}

We thank the department of Surgical Oncology of First Hospital of China Medical University for technical assistance. The corresponding author had full access to all the data and analyses.

\section{Financial \& competing interests disclosure}

This manuscript was supported by funding from Doctoral Scientific Research Startup Foundation of Liaoning Province (20170520442), National Key R\&D Program of China (MOST-2016YFC1303200) (MOST-2016YFC1303202) (MOST2017YFC0908300) (MOST-2017YFC0908305) and Program for Liaoning Innovative Research Team in University (LT2016005). The authors have no other relevant affiliations or financial involvement with any organization or entity with a financial interest in or financial conflict with the subject matter or materials discussed in the manuscript apart from those disclosed.

No writing assistance was utilized in the production of this manuscript.

\section{Open access}

This work is licensed under the Attribution-NonCommercial-NoDerivatives 4.0 Unported License. To view a copy of this license, visit http://creativecommons.org/licenses/by-nc-nd/4.0/

\section{References}

Papers of special note have been highlighted as: $\bullet \bullet$ of considerable interest

1. Chen DS, Mellman I. Elements of cancer immunity and the cancer-immune set point. Nature 541(7637), 321-330 (2017).

2. Sharma P, Allison JP. Immune checkpoint targeting in cancer therapy: toward combination strategies with curative potential. Cell 161(2), 205-214 (2015).

3. Elias R, Morales J, Rehman Y, Khurshid H. Immune checkpoint inhibitors in older adults. Curr. Oncol. Rep. 18(8), 47 (2016).

4. National Cancer Institute. The Surveillance, Epidemiology and EndResults. https://seer.cancer.gov/ (2020).

5. Hurria A, Levit LA, Dale W et al. Improving the evidence base for treating older adults with cancer: american society of clinical oncology statement. J. Clin. Oncol. 33(32), 3826-3833 (2015).

6. Pawelec G, Derhovanessian E, Larbi A. Immunosenescence and cancer. Crit. Rev. Oncol. Hematol. 75(2), 165-172 (2010).

7. Franceschi C, Bonafe M, Valensin S. Human immunosenescence: the prevailing of innate immunity, the failing of clonotypic immunity and the filling of immunological space. Vaccine 18(16), 1717-1720 (2000).

8. Tomihara K, Curiel TJ, Zhang B. Optimization of immunotherapy in elderly cancer patients. Crit. Rev. Oncog. 18(6), 573-583 (2013).

9. Wu Q, Wang Q, Tang X et al. Correlation between patients' age and cancer immunotherapy efficacy. Oncoimmunology 8(4), e1568810 (2019).

10. Li J, Gu J. Efficacy of immune checkpoint inhibitors in cancer patients of different ages: a meta-analysis. Future. Oncol. 15(31), 3633-3646 (2019).

11. Li P, Yang X, Feng Y et al. The impact of immunosenescence on the efficacy of immune checkpoint inhibitors in melanoma patients: a meta-analysis. Onco. Targets Ther. 11, 7521-7527 (2018).

12. Jadad AR, Moore RA, Carroll D et al. Assessing the quality of reports of randomized clinical trials: is blinding necessary? Control. Clin. Trials 17(1), 1-12 (1996).

13. Schmidt FL, Oh IS, Hayes TL. Fixed- versus random-effects models in meta-analysis: model properties and an empirical comparison of differences in results. Br. J. Math. Stat. Psychol. 62(Pt 1), 97-128 (2009).

14. Higgins JP, Thompson SG, Deeks JJ, Altman DG. Measuring inconsistency in meta-analyses. BMJ 27(7414), 557-560 (2003).

15. Egger M, Davey Smith G, Schneider M, Minder C. Bias in meta-analysis detected by a simple, graphical test. BMJ 315(7109), 629-634 (1997).

16. Begg CB, Mazumdar M. Operating characteristics of a rank correlation test for publication bias. Biometrics 50(4), 1088-1101 (1994).

17. Fisher DJ, Carpenter JR, Morris TP, Freeman SC, Tierney JF. Meta-analytical methods to identify who benefits most from treatments: daft, deluded, or deft approach? BMJ 356, j573 (2017).

18. Socinski MA, Jotte RM, Cappuzzo F et al. Atezolizumab for first-line treatment of metastatic nonsquamous NSCLC. N. Engl. J. Med. 378(24), 2288-2301 (2018). 
-• Phase III study demonstrates atezolizumab plus chemotherapy as first-line treatment for metastatic nonsquamous non-small-cell lung cancer (NSCLC) regardless of PD-L1 expression and EGFR or ALK genetic alteration status.

19. Shitara K, Ozguroglu M, Bang YJ et al. Pembrolizumab versus paclitaxel for previously treated, advanced gastric or gastro-oesophageal junction cancer (KEYNOTE-061): a randomised, open-label, controlled, Phase III trial. Lancet 392(10142), 123-133 (2018).

20. Schmid P, Adams S, Rugo HS et al. Atezolizumab and nab-paclitaxel in advanced triple-negative breast cancer. N. Engl. J. Med. 379(22), 2108-2121 (2018).

21. Paz-Ares L, Luft A, Vicente D et al. Pembrolizumab plus chemotherapy for squamous non-small-cell lung cancer. N. Engl. J. Med. 379(21), 2040-2051 (2018).

-. Phase III study demonstrates pembrolizumab plus chemotherapy as first-line treatment for metastatic squamous NSCLC regardless of PD-L1 expression.

22. Motzer RJ, Tannir NM, Mcdermott DF et al. Nivolumab plus ipilimumab versus sunitinib in advanced renal-cell carcinoma. N. Engl. J. Med. 378(14), 1277-1290 (2018).

-• Phase III study demonstrates nivolumab plus ipilimumab as first-line treatment for intermediate- or poor-risk advanced clear-cell renal-cell carcinoma.

23. Larkin J, Minor D, D'angelo $S$ et al. Overall survival in patients with advanced melanoma who received nivolumab versus investigator's choice chemotherapy in CheckMate 037: a randomized, controlled, open-label Phase III trial. J. Clin. Oncol. 36(4), 383-390 (2018).

24. Horn L, Mansfield AS, Szczesna A et al. First-line atezolizumab plus chemotherapy in extensive-stage small-cell lung cancer. $N$. Engl. J. Med. 379(23), 2220-2229 (2018).

-. Phase III study demonstrates atezolizumab plus chemotherapy as first-line treatment for extensive-stage small-cell lung cancer.

25. Hellmann MD, Ciuleanu TE, Pluzanski A et al. Nivolumab plus ipilimumab in lung cancer with a high tumor mutational burden. $N$. Engl. J. Med. 378(22), 2093-2104 (2018).

26. Gandhi L, Rodriguez-Abreu D, Gadgeel S et al. Pembrolizumab plus chemotherapy in metastatic non-small-cell lung cancer. N. Engl. J. Med. 378(22), 2078-2092 (2018).

27. Eggermont AMM, Blank CU, Mandala M, Long GV. Adjuvant pembrolizumab versus placebo in resected stage iii melanoma. $N$. Engl. J. Med. 378(19), 1789-1801 (2018).

-• Phase III study demonstrates pembrolizumab as adjuvant therapy for resected, high-risk stage III melanoma.

28. Cohen EEW, Soulieres D, Le Tourneau C et al. Pembrolizumab versus methotrexate, docetaxel, or cetuximab for recurrent or metastatic head-and-neck squamous cell carcinoma (KEYNOTE-040): a randomised, open-label, Phase III study. Lancet 393(10167), 156-167 (2018).

29. Barlesi F, Vansteenkiste J, Spigel D et al. Avelumab versus docetaxel in patients with platinum-treated advanced non-small-cell lung cancer (JAVELIN Lung 200): an open-label, randomised, Phase III study. Lancet Oncol. 19(11), 1468-1479 (2018).

30. Bang YJ, Ruiz EY, Van Cutsem E et al. Phase III, randomised trial of avelumab versus physician's choice of chemotherapy as third-line treatment of patients with advanced gastric or gastro-oesophageal junction cancer: primary analysis of JAVELIN Gastric 300. Ann. Oncol. 29(10), 2052-2060 (2018).

31. Antonia SJ, Villegas A, Daniel D et al. Overall survival with durvalumab after chemoradiotherapy in stage III NSCLC. N. Engl. J. Med. 379(24), 2342-2350 (2018).

-• Phase III study demonstrates a survival advantage with durvalumab after concurrent chemoradiation therapy in unresectable stage III NSCLC

32. Rittmeyer A, Barlesi F, Waterkamp D et al. Atezolizumab versus docetaxel in patients with previously treated non-small-cell lung cancer (OAK): a Phase III, open-label, multicentre randomised controlled trial. Lancet 389(10066), 255-265 (2017).

33. Maio M, Scherpereel A, Calabro L et al. Tremelimumab as second-line or third-line treatment in relapsed malignant mesothelioma (DETERMINE): a multicentre, international, randomised, double-blind, placebo-controlled Phase IIb trial. Lancet Oncol. 18(9), 1261-1273 (2017).

34. Kang YK, Boku N, Satoh T et al. Nivolumab in patients with advanced gastric or gastro-oesophageal junction cancer refractory to, or intolerant of, at least two previous chemotherapy regimens (ONO-4538-12, ATTRACTION-2): a randomised, double-blind, placebo-controlled, Phase III trial. Lancet 390(10111), 2461-2471 (2017).

35. Govindan R, Szczesna A, Ahn MJ et al. Phase III trial of ipilimumab combined with paclitaxel and carboplatin in advanced squamous non-small-cell lung cancer. J. Clin. Oncol. 35(30), 3449-3457 (2017).

36. Carbone DP, Reck M, Paz-Ares L et al. First-line nivolumab in stage IV or recurrent non-small-cell lung cancer. N. Engl. J. Med. 376(25), 2415-2426 (2017).

37. Bellmunt J, De Wit R, Vaughn DJ et al. Pembrolizumab as second-line therapy for advanced urothelial carcinoma. N. Engl. J. Med. 376(11), 1015-1026 (2017).

38. Beer TM, Kwon ED, Drake CG et al. Randomized, double-blind, Phase III trial of ipilimumab versus placebo in asymptomatic or minimally symptomatic patients with metastatic chemotherapy-naive castration-resistant prostate cancer. J. Clin. Oncol. 35(1), 40-47 (2017). 
39. Reck M, Rodriguez-Abreu D, Robinson AG et al. Pembrolizumab versus chemotherapy for PD-L1-positive non-small-cell lung cancer. N. Engl. J. Med. 375(19), 1823-1833 (2016).

40. Reck M, Luft A, Szczesna A et al. Phase III randomized trial of ipilimumab plus etoposide and platinum versus placebo plus etoposide and platinum in extensive-stage small-cell lung cancer. J. Clin. Oncol. 34(31), 3740-3748 (2016).

41. Herbst RS, Baas P, Kim DW et al. Pembrolizumab versus docetaxel for previously treated, PD-L1-positive, advanced non-small-cell lung cancer (KEYNOTE-010): a randomised controlled trial. Lancet 387(10027), 1540-1550 (2016).

42. Ferris RL, Blumenschein G Jr, Fayette J et al. Nivolumab for recurrent squamous-cell carcinoma of the head and neck. N. Engl. J. Med. 375(19), 1856-1867 (2016).

43. Robert C, Long GV, Brady B et al. Nivolumab in previously untreated melanoma without BRAF mutation. N. Engl. J. Med. 372(4), 320-330 (2015).

-• Phase III study demonstrates nivolumab monotherapy for untreated metastatic melanoma without a BRAF mutation.

44. Ribas A, Puzanov I, Dummer R et al. Pembrolizumab versus investigator-choice chemotherapy for ipilimumab-refractory melanoma (KEYNOTE-002): a randomised, controlled, Phase II trial. Lancet Oncol. 16(8), 908-918 (2015).

45. Motzer RJ, Escudier B, Mcdermott DF et al. Nivolumab versus everolimus in advanced renal-cell carcinoma. N. Engl. J. Med. 373(19), 1803-1813 (2015).

46. Brahmer J, Reckamp KL, Baas P et al. Nivolumab versus docetaxel in advanced squamous-cell non-small-cell lung cancer. N. Engl. J. Med. 373(2), 123-135 (2015).

47. Borghaei H, Paz-Ares L, Horn L et al. Nivolumab versus docetaxel in advanced nonsquamous non-small-cell lung cancer. N. Engl. J. Med. 373(17), 1627-1639 (2015).

48. Kwon ED, Drake CG, Scher HI et al. Ipilimumab versus placebo after radiotherapy in patients with metastatic castration-resistant prostate cancer that had progressed after docetaxel chemotherapy (CA184-043): a multicentre, randomised, double-blind, Phase III trial. Lancet Oncol. 15(7), 700-712 (2014).

49. Ribas A, Kefford R, Marshall MA et al. Phase III randomized clinical trial comparing tremelimumab with standard-of-care chemotherapy in patients with advanced melanoma. J. Clin. Oncol. 31(5), 616-622 (2013).

50. Robert C, Thomas L, Bondarenko I et al. Ipilimumab plus dacarbazine for previously untreated metastatic melanoma. N. Engl. J. Med. 364(26), 2517-2526 (2011).

51. Hodi FS, O'day SJ, Mcdermott DF et al. Improved survival with ipilimumab in patients with metastatic melanoma. $N$. Engl. J. Med. 363(8), 711-723 (2010).

52. Hoos A. Development of immuno-oncology drugs from CTLA4 to PD1 to the next generations. Nat. Rev. Drug Discov. 15(4), 235-247 (2016).

53. Tumeh PC, Harview CL, Yearley JH et al. PD-1 blockade induces responses by inhibiting adaptive immune resistance. Nature 515(7528), 568-571 (2014).

54. Dong H, Strome SE, Salomao DR et al. Tumor-associated B7-H1 promotes T-cell apoptosis: a potential mechanism of immune evasion. Nat. Med. 8(8), 793-800 (2002).

55. Palmer S, Albergante L, Blackburn CC, Newman TJ. Thymic involution and rising disease incidence with age. Proc. Natl Acad. Sci. USA 115(8), 1883-1888 (2018).

56. Kugel CH 3rd, Douglass SM, Webster MR et al. Age correlates with response to anti-PD1, reflecting age-related differences in intratumoral effector and regulatory T-cell populations. Clin. Cancer. Res. 24(21), 5347-5356 (2018).

57. Extermann M, Hurria A. Comprehensive geriatric assessment for older patients with cancer. J. Clin. Oncol. 25(14), 1824-1831 (2007).

58. Shahir MA, Lemmens VE, Van De Poll-Franse LV, Voogd AC, Martijn H, Janssen-Heijnen ML. Elderly patients with rectal cancer have a higher risk of treatment-related complications and a poorer prognosis than younger patients: a population-based study. Eur. J. Cancer 42(17), 3015-3021 (2006).

59. Zhao $S$, Ren $S$, Jiang $T$ et al. Low-dose apatinib optimizes tumor microenvironment and potentiates antitumor effect of PD-1/PD-L1 blockade in lung cancer. Cancer Immunol. Res. 7(4), 630-643 (2019).

60. Gotwals P, Cameron S, Cipolletta D et al. Prospects for combining targeted and conventional cancer therapy with immunotherapy. Nat. Rev. Cancer 17(5), 286-301 (2017).

61. Lee KWC, Lord SJ, Kasherman L et al. The impact of smoking on the effectiveness of immune checkpoint inhibitors - a systematic review and meta-analysis. Acta Oncol. doi:10.1080/0284186X.2019.1670354 (2019) (Epub ahead of print).

62. Abdel-Rahman O. Smoking and EGFR status may predict outcomes of advanced NSCLC treated with PD-(L)1 inhibitors beyond first line: a meta-analysis. Clin. Respir. J. 12(5), 1809-1819 (2018). 
